\title{
INFLUÊNCIA DE FATORES CLIMÁTICOS E OPERACIONAIS SOBRE A UNIFORMIDADE DE DISTRIBUIÇÃO DE ÁGUA, EM UM SISTEMA DE IRRIGAÇÃO POR ASPERSÃO DE ALTA PRESSÃO ${ }^{1}$
}

\author{
Hamilton Jorge Azevedo², Salassier Bernardo ${ }^{3}$, Marcio Mota Ramos ${ }^{4}$, \\ Gilberto Chohaku Sediyama ${ }^{4} \&$ Paulo Roberto Cecon ${ }^{5}$ \\ RESUMO
}

\begin{abstract}
Realizou-se um estudo para se avaliar a uniformidade de distribuição de água em um sistema de irrigação por aspersão de alta pressão, na região norte-fluminense, RJ. Por meio da metodologia descrita pela ASAE (1990) foi avaliada a uniformidade de distribuição de água utilizando-se, para isso, o coeficiente de uniformidade de Christiansen (CUC) com aspersores em disposição retangular e triangular. Para cada uma das situações, o CUC foi analisado em função das seguintes variáveis independentes: velocidade e direção do vento, pressão de operação do aspersor, espaçamento entre aspersores na linha lateral, espaçamento entre linhas laterais e velocidade de rotação do aspersor. A análise dos dados foi feita por meio de regressão múltipla, gerando-se modelos estatísticos para a estimativa do CUC. Observou-se que o aumento da velocidade do vento e do espaçamento entre aspersores diminui o CUC, enquanto o aumento da pressão de operação do aspersor o elevou nos intervalos estudados.
\end{abstract}

Palavras-chave: aspersão, uniformidade, vento, clima

\section{INFLUENCE OF CLIMATIC AND OPERATIONAL FACTORS ON THE UNIFORMITY OF WATER DISTRIBUTION IN A HIGH PRESSURE SPRINKLE SYSTEM}

\begin{abstract}
A study was performed to evaluate the water distribution uniformity in a high pressure sprinkle system in the norte-fluminense region, RJ. The field experiment was carried out according to ASAE (1990) methodology. Christiansen uniformity coefficient (CUC) was used to evaluate the water application distribution uniformity, with sprinklers placed in rectangular and triangular spacings. For each one of these situations the CUC was analysed according to the following independent variables: wind speed and direction, operation pressure, spacings between sprinklers and lateral lines, and sprinkler speed rotation. The evaluation of data was made with multiple regression analysis, which made it possible to develop a statistical model to estimate the CUC. It was observed that the increase in wind speed and spacing between sprinklers reduced the CUC, while the increase in sprinkler pressure increased the CUC.
\end{abstract}

Key words: sprinkle, uniformity, wind, weather

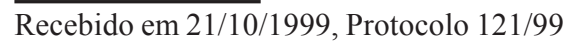

${ }^{1}$ Trabalho financiado pela Fundação do Banco do Brasil, com apoio da usina Sapucaia S.A.

${ }^{2}$ Eng. Agrônomo, D.Sc., Campus Dr. Leonel Miranda UFRRJ, Estrada do Açúcar s/n, km 5, CEP 28020 - 560, Campos dos Goytacazes, RJ. Fone: (0xx24) 733 0505, Fax: (0xx24) 723 1324. E-mail: clmufrrj@rol.com.br

${ }^{3}$ Professor Titular, CCTA, UENF, CEP 28015 - 620, Campos dos Goytacazes, RJ. Fone: (0xx24) 726 3752, Fax: (0xx24) 7263711. E-mail: salassie@uenf.br

${ }^{4}$ Prof. Titular, Depto. Engenharia Agrícola, UFV, CEP 36570 - 000, Viçosa, MG. Fone: (0xx31)899 1914/899 1905, Fax: (0xx31)899 2735.E-mail:mmramos@mail.ufv.br, sediyama@mail.ufv.br

${ }^{5}$ Prof. Adjunto, Depto. de Estatística e Informática, UFV, CEP 36570 - 000, Viçosa, MG. Fone: (0xx31) 899 1781, Fax: (0xx31) 8992314. E-mail:cecon@dpi.ufv.br 


\section{INTRODUÇÃO}

A irrigação por aspersão tem por objetivo distribuir água sobre a superfície do solo, de modo a permitir a sua infiltração sem escoamento superficial; além disso, a água deve ser distribuída de maneira o mais uniforme possível, de forma a se obter uma adequada uniformidade de aplicação em condições de campo. Muitos fatores podem interferir no nível de uniformidade de distribuição da água, como espaçamento entre aspersores, velocidade e direção do vento, pressão de operação dos aspersores, velocidade e uniformidade de rotação dos aspersores, entre outros (Christiansen, 1941).

A irrigação por aspersão é muito influenciada pela ação do vento e vários trabalhos de pesquisa têm demonstrado que o aumento da velocidade do vento provoca diminuição da uniformidade de distribuição de água, como resultado da distorção do perfil de aplicação de água e da redução da área coberta pelo aspersor e, consequentemente, pela diminuição da sobreposição dos jatos de água (Gomide et al., 1980; Ribeiro, 1982; Vories \& Bernuth, 1986; Paz, 1990; Martim-Benito et al., 1992; Alves \& Castro, 1995).

A influência da direção do vento sobre a distribuição de água do aspersor é bastante controversa na literatura. Alguns trabalhos têm relatado que o efeito encontrado sobre o CUC foi muito pequeno, ou nulo (Wiersma, 1955; Nogueira \& Souza, 1987). Ptacek em 1972 citado por Moynahan (1972) verificou não existir orientação única da linha lateral em relação ao vento, que possa resultar em maior CUC; outros autores afirmaram que o melhor posicionamento da linha lateral é perpendicular à direção do vento (Shull \& Dylla, 1976b; Ribeiro, 1983; Bernardo, 1995); finalmente, que o melhor posicionamento da linha lateral é paralelo à direção do vento (Vories \& Bernuth, 1986).

Poucos estudos têm sido realizados para quantificar os efeitos da velocidade de rotação do aspersor sobre a uniformidade de distribuição de água. Os trabalhos pioneiros têm evidenciado efeito de diminuição do raio de alcance do jato, aumento na pulverização do jato de água e, geralmente, uniformidade de distribuição ruim quando a velocidade de rotação do aspersor aumenta (Christiansen, 1942; Bilanski \& Kider, 1958, citado por Moynahan, 1972). A velocidade de rotação adequada do aspersor, segundo Keller (1984) é aquela cuja velocidade periférica do jato na área molhada é de $1,5 \mathrm{~m} \mathrm{~s}^{-1}$; assim, em um aspersor do tipo canhão com diâmetro de alcance de $140 \mathrm{~m}$, deve ocorrer uma revolução a cada 4,9 min.

O aumento da pressão de serviço do aspersor, dentro de certo limite de valores, produz aumento na uniformidade de distribuição de água (Wiersma, 1955; Vories \& Bernuth, 1986; Carvalho 1991; Martim-Benito et al., 1992; Zocoler et al., 1995). A partir de certo limite, o aumento da pressão de serviço provoca excesso de pulverização do jato, ocasionando diminuição do raio de alcance e, para dado espaçamento entre aspersores, redução da uniformidade de distribuição de água (Christiansen, 1942; Shull \& Dylla, 1976b; Charmelo, 1990; Zocoler et al., 1995).

Em geral, quando o espaçamento entre aspersores aumenta, a uniformidade de distribuição diminui; entretanto, a combinação de outros fatores deve ser considerada na seleção do melhor espaçamento, como pressão de operação, diâmetro do bocal, condições de vento, raio de alcance do jato, custos etc.
Este trabalho teve por objetivo estudar a influência da velocidade e da direção do vento, da pressão de operação do aspersor, da velocidade de rotação do aspersor, do espaçamento entre aspersores na linha lateral, do espaçamento entre linhas laterais e das disposições retangular e triangular do sistema, sobre a uniformidade de distribuição de água de um aspersor de alta pressão.

\section{MATERIAL E MÉTODOS}

Este trabalho foi conduzido na fazenda de propriedade da usina Sapucaia S.A, no município de Campos dos Goytacazes, RJ. O local é plano e o solo sem cobertura vegetal, latitude de $21^{\circ} 39^{\prime} 22^{\prime \prime} \mathrm{S}$, longitude de $41^{\circ} 24^{\prime} 01^{\prime \prime} \mathrm{W}$ e altitude de $10,9 \mathrm{~m}$, numa área de cerca de 4,0 ha.

Os testes foram realizados com aspersor canhão rotativo de impacto, modelo HS 244 B, com diâmetro dos bocais de 35,4 por $10,8 \mathrm{~mm}$ e ângulo de inclinação do jato de $24^{\circ}$; o bocal de saída principal do aspersor operou durante os testes a uma altura de $1,05 \mathrm{~m}$ da superfície do solo, enquanto a vazão do aspersor para cada teste foi determinada por meio de um hidrômetro de turbina horizontal de $150 \mathrm{~mm}$ de diâmetro e dentro do intervalo das vazões trabalhadas $\left(95,2\right.$ a $\left.142,9 \mathrm{~m}^{3} \mathrm{~h}^{-1}\right)$; os erros de leitura do hidrômetro, segundo sua curva característica de desempenho fornecida pelo fabricante, foram iguais ou inferiores a $0,3 \%$.

A pressão de serviço do aspersor foi determinada no jato do bocal principal, por meio de um manômetro acoplado a um tubo de pitot, realizada imediatamente antes de cada teste. Para monitoramento da pressão durante a realização dos testes, usou-se o manômetro acoplado à base do aspersor, enquanto os testes foram realizados com um único aspersor trabalhando em pressões que variaram de 318 a $729 \mathrm{kPa}$, ajustadas por meio de duas válvulas borboleta de $100 \mathrm{~mm}$, sendo uma instalada na saída do conjunto moto-bomba e outra no tubo de subida do aspersor.

A metodologia usada foi aquela descrita pela ASAE (1990) e o tempo de operação durante cada teste de aspersor foi de pelo menos 60 min; a disposição dos pluviômetros para a coleta de água aplicada pelo aspersor foi posicionada numa malha quadrada de $6 \times 6 \mathrm{~m}$.

Utilizaram-se 1156 pluviômetros para coletar a água distribuída pelo aspersor e a medição da água foi realizada com provetas de 500 e $50 \mathrm{~mL}$ de capacidade, usadas conforme o volume coletado.

Os pluviômetros utilizados foram construídos de folha de flandres, com formato cilíndrico, $10 \mathrm{~cm}$ de diâmetro e $15 \mathrm{~cm}$ de altura, apoiados sobre tijolos previamente nivelados junto ao solo.

Os dados climáticos usados neste trabalho foram obtidos de uma estação climatológica existente ao lado da área de ensaio e constavam, entre outros, dos seguintes instrumentos: anemógrafo e catavento, instalados a 2,0 $\mathrm{m}$ da superfície do solo; o anemógrafo tinha capacidade de operação diária e registrava o vento a cada $500 \mathrm{~m}$ de deslocamento de ar. Foram realizadas leituras da direção do vento, em graus, a cada cinco minutos de teste, sendo observados, a cada leitura, os valores máximo e mínimo da direção do vento, por meio dos quais se determinou a direção média do vento. 
Antes de se iniciar cada teste, os pluviômetros de um setor radial de abrangência do jato eram virados para baixo, de forma que o jato pudesse permanecer nesse setor para a medição da pressão de serviço do aspersor; após essa medição e a leitura inicial do hidrômetro, iniciava-se o teste, colocando-se o aspersor na posição de partida e acionando-se o cronômetro de controle de duração do teste; a seguir, desviravam-se rapidamente os pluviômetros, antes que fossem atingidos pelo jato do aspersor; por fim, completava-se o tempo mínimo de 60 min para finalizar o teste, momento em que se desligava o conjunto moto-bomba e se fazia a leitura final do hidrômetro.

Para se avaliar a evaporação de água ocorrida durante a leitura dos pluviômetros, antes do início de cada teste, foram instalados oito pluviômetros iguais aos utilizados para coleta da água dos aspersores, nos quais eram colocados $50 \mathrm{~mL}$ de água, tampados e posicionados junto da área de teste. Após o final do teste do aspersor, os oito pluviômetros eram destampados e o volume de quatro deles lido com auxílio de uma proveta de $50 \mathrm{~mL}$; depois, eram feitas as leituras dos pluviômetros que coletaram a água distribuída pelo aspersor e, ao final dessas leituras, eram lidos os quatro pluviômetros restantes; desta forma, obteve-se o volume inicial e final dos pluviômetros para cada ensaio e a evaporação média.

Durante a leitura dos pluviômetros de cada ensaio foi anotado o tempo do início e do final de leitura de cada linha de pluviômetros. Com as informações mencionadas e com as equações de calibração das provetas, realizou-se a correção ponderada da evaporação da água ocorrida durante a leitura de cada linha de pluviômetros.

Estimou-se a uniformidade de distribuição de água por meio do coeficiente de uniformidade de Christiansen (CUC) expressa pela seguinte equação:

$$
\mathrm{CUC}=100\left(1-\frac{\sum_{\mathrm{i}=1}^{\mathrm{n}}\left|\mathrm{x}_{\mathrm{i}}-\overline{\mathrm{x}}\right|}{\sum_{\mathrm{i}=1}^{\mathrm{n}} \mathrm{x}_{\mathrm{i}}}\right)
$$

em que:

$\overline{\mathrm{X}}$ - precipitação média dos pluviômetros

$\mathrm{X}_{\mathrm{i}}$ - precipitação observada em cada pluviômetro

Para se simular a sobreposição dos diferentes espaçamentos entre aspersores, nas disposições retangular e triangular e se calcular os CUC desenvolveu-se um aplicativo computacional, por meio do qual foram simulados 81 diferentes espaçamentos entre aspersores para as disposições retangular e triangular, com espaçamentos de 48 x 48 m a 96 x 96 m, variando de 6 em 6 m.

Realizou-se análise de regressão múltipla do CUC em função das variáveis independentes velocidade do vento (v), direção do vento em relação à linha lateral (DV), pressão de serviço do $\operatorname{aspersor}(p)$, espaçamento entre aspersores na linha lateral $(\mathrm{Ea})$, espaçamento entre linhas laterais (El) e velocidade de rotação dos aspersores (VRA).

\section{RESULTADOS E DISCUSSÃO}

Nesse estudo foram realizados 114 ensaios de campo para a determinação dos coeficientes de uniformidade de distribuição de água, os quais foram analisados, em função da velocidade e da direção do vento em relação à linha lateral, pressão de operação do aspersor, espaçamento entre aspersores na linha lateral, espaçamento entre linhas laterais e velocidade de rotação dos aspersores. Na Tabela 1 são apresentados os resumos das análises dessas variáveis.

Tabela 1. Resumo das análises das variáveis independentes velocidade do vento $(v)$, direção do vento em relação à linha lateral (DV), pressão de operação do aspersor (p), espaçamento entre aspersores na linha lateral (Ea), espaçamento entre linhas laterais (El) e velocidade de rotação do aspersor (VRA)

\begin{tabular}{lcccccc}
\hline & \multicolumn{7}{c}{ Variáveis } \\
\cline { 2 - 7 } Parâmetros & $\begin{array}{c}\mathrm{v} \\
\mathrm{m} \mathrm{s}^{-1}\end{array}$ & $\begin{array}{c}\mathrm{DV} \\
\text { graus }\end{array}$ & $\begin{array}{c}\mathrm{p} \\
\mathrm{kPa}\end{array}$ & $\begin{array}{c}\mathrm{Ea} \\
\mathrm{m}\end{array}$ & $\begin{array}{c}\mathrm{El} \\
\mathrm{m}\end{array}$ & $\begin{array}{c}\text { VRA } \\
\mathrm{rpm}\end{array}$ \\
\hline Média & 3,13 & 93,23 & 560,8 & 63,12 & 80,88 & 0,28 \\
Desvio-padrão & 1,50 & 51,21 & 107,06 & 12,68 & 12,70 & 0,13 \\
Mínimo & 0,53 & 1 & 318 & 48 & 48 & 0,09 \\
Máximo & 7,17 & 179 & 729 & 96 & 96 & 0,83 \\
Observações & 9234 & 9234 & 9234 & 9234 & 9234 & 9234 \\
\hline
\end{tabular}

\section{Distribuição retangular}

$$
\begin{gathered}
\mathrm{CUC}=-28,105447+14,726691 \mathrm{v}+0,061102 \mathrm{DV}+0,097540 \mathrm{p}+ \\
0,842989 \mathrm{Ea}+1,067157 \mathrm{El}+17,280424 \mathrm{VRA}-0,408547 \mathrm{v}^{2}- \\
0,000436 \mathrm{DV}^{2}-0,000046 \mathrm{p}^{2}-0,006499 \mathrm{Ea}^{2}-0,006906 \mathrm{El}^{2}- \\
20,255217 \mathrm{VRA}^{2}-0,089418 \mathrm{vEa}-0,163169 \mathrm{vEl}\left(\mathrm{R}^{2}=0,90\right)
\end{gathered}
$$

\section{Distribuição triangular}

$\mathrm{CUC}=-24,455095+12,261574 \mathrm{v}-0,23780 \mathrm{DV}+0,094294 \mathrm{p}+$
$1,037286 \mathrm{Ea}+0,915146 \mathrm{El}+11,145366 \mathrm{VRA}-0,432102 \mathrm{v}^{2}+$
$0,00010 \mathrm{DV}^{2}-0,000043 \mathrm{p}^{2}-0,007529 \mathrm{Ea}^{2}-0,006422 \mathrm{El}^{2}-$
$14,578673 \mathrm{VRA}^{2}-0,125032 \mathrm{vEa}-0,088311 \mathrm{vEl}\left(\mathrm{R}^{2}=0,84\right)$

As Eqs. 2 e 3 são os modelos estatísticos obtidos pela análise de regressão e descrevem o coeficiente de uniformidade de Christiansen (CUC) em função das variáveis estudadas, nas disposições retangular e triangular, respectivamente; para isto, foram analisadas 9234 linhas de observação.

Os coeficientes das Eqs. (2) e (3) foram significativos a 1\% de probabilidade pelo teste " $t$ ". Observa-se, em ambas as equações, que todas as variáveis estudadas tiveram efeito quadrático sobre o CUC.

\section{Aspersores em disposição retangular}

Velocidade de rotação do aspersor: A velocidade de rotação do aspersor (VRA) é, segundo Christiansen (1942) um dos fatores que interferem na uniformidade de distribuição de água; entretanto, não se encontraram estudos indicando a variação da velocidade de rotação do aspersor, desde que, na prática, não prejudique a uniformidade de distribuição de água. Neste trabalho foram utilizados dados num intervalo de 0,09 a 0,83 rpm, o que representa uma revolução a cada 11,1 e 1,2 min, respectivamente.

Na Figura 1 são apresentadas as estimativas da variação do CUC em função da velocidade de rotação do aspersor (VRA) para diferentes velocidades do vento (v), Ea de $72 \mathrm{~m}$, El de $84 \mathrm{~m}$, $\mathrm{DV}$ de $90^{\circ}$ e p de $686 \mathrm{kPa}$. Dentro dos intervalos de valores apresentados, o CUC variou de 85 a $30 \%$ quando v e VRA 
variaram de $1,0 \mathrm{~m} \mathrm{~s}^{-1}$ e $0,43 \mathrm{rpm}$ a $7,0 \mathrm{~m} \mathrm{~s}^{-1}$ e $0,8 \mathrm{rpm}$, respectivamente. O VRA apresentou efeito quadrático em relação ao CUC, cujo valor máximo foi atingido quando VRA foi de $0,43 \mathrm{rpm}$, o que eqüivale a uma revolução do aspersor a cada 2,3 min. Este resultado difere de Keller (1984) que recomenda, para esse tipo de aspersor, uma revolução a cada 4,9 min. Observa-se, também, que na faixa de variação apresentada de VRA, o seu efeito sobre o CUC foi muito pequeno, apresentando variação máxima de $2,8 \%$, para dada velocidade de vento; com relação à velocidade do vento, verificou-se que o valor médio do CUC reduziu de $49 \%$ quando v aumentou de 1 para $7 \mathrm{~m} \mathrm{~s}^{-1}$.

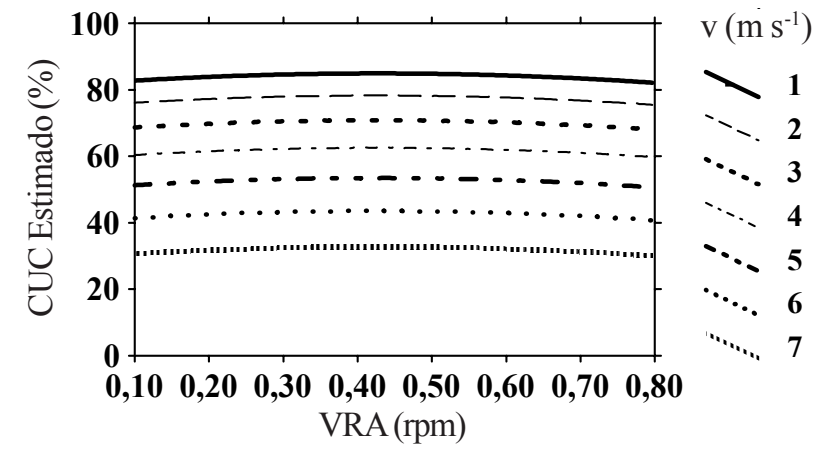

Figura 1. Estimativa do coeficiente de uniformidade de Christiansen em função da velocidade de rotação do aspersor para diferentes velocidades do vento, Ea de $72 \mathrm{~m}$, El de $84 \mathrm{~m}, \mathrm{DV}$ de $90^{\circ}$ e p de $686 \mathrm{kPa}$, com distribuição entre aspersores retangulares

Direção do vento em relação à linha lateral: A direção do vento em relação à linha lateral (DV) é, segundo Shull e Dylla (1976a), Ribeiro (1983) e Bernardo (1995) um dos fatores que interferem na distribuição de água dos aspersores; assim, nos diferentes testes de aspersores realizados no campo, foram observados valores do sentido do vento dentro do intervalo de 1 a $359^{\circ}$; entretanto para transformar os valores de sentido do vento em direção do vento, reduziram-se todos os valores maiores que $180^{\circ}$, pela subtração de cada um deles em $180^{\circ}$; desta maneira, todos os valores passaram a representar a direção do vento compreendida entre 1 e $179^{\circ}$, o que foi feito para a confirmação de que nesse intervalo o CUC pode ser descrito em relação ao DV, por um polinômio de segundo grau, tendo em vista a recomendação da literatura em posicionar a linha lateral em relação ao vento em $90^{\circ}$.

Na Figura 2 tem-se as estimativas do CUC em função da direção do vento para diferentes velocidades, Ea de $72 \mathrm{~m}$, El de $84 \mathrm{~m}$, p de $686 \mathrm{kPa}$ e VRA de 0,43 rpm. O CUC estimado apresentou um máximo quando DV foi igual a $70^{\circ}$ para qualquer condição de velocidade do vento e o valor máximo de CUC para DV, de $70^{\circ}$, parece ratificar a recomendação da literatura de se posicionar a linha lateral em $90^{\circ}$ com relação à direção do vento, tendo em vista a natureza experimental dos dados. A diferença do CUC estimado entre DV de $70^{\circ}$ e $90^{\circ}$ foi de $0,17 \%$, ou seja, valor insignificante em se tratando de valores de CUC. É importante frisar que a influência do DV sobre o CUC não foi expressiva, apresentando diferença média entre valores extremos, em torno de $4,0 \%$, para uma velocidade do vento.

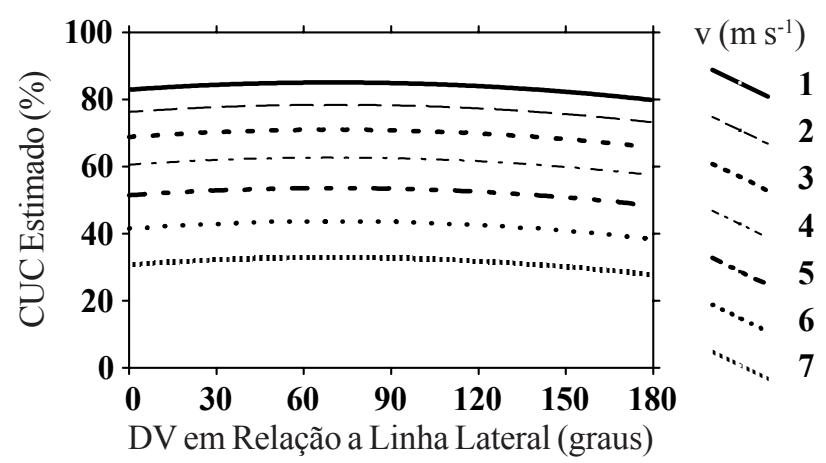

Figura 2. Estimativa do coeficiente de uniformidade de Christiansen em função da direção do vento em relação à linha lateral, para diferentes velocidades do vento, Ea de $72 \mathrm{~m}$, El de 84 m, VRA de $0,43 \mathrm{rpm}$ e p de $686 \mathrm{kPa}$, com distribuição entre aspersores retangulares

Pressão de operação do aspersor: A uniformidade de distribuição de água é afetada pela pressão de operação do aspersor e existe uma pressão ideal para cada aspersor, a fim de garantir um raio de alcance e pulverização do jato adequados (Chistiansen, 1942; Wiersma, 1955; Shull \& Dylla, 1976 b; Ali \& Barefoot, 1981; Arruda, 1981; Charmelo, 1990).

Neste trabalho, a influência da pressão de operação sobre o CUC foi estudada num intervalo compreendido entre 318 e 729 $\mathrm{kPa}$. Na Figura 3 são apresentadas as estimativas do CUC em função da pressão de operação do aspersor para diferentes velocidades do vento, Ea de $72 \mathrm{~m}$, El de $84 \mathrm{~m}$, DV de $90^{\circ}$ e VRA de $0,43 \mathrm{rpm}$. Observa-se, nesta figura, que o CUC aumentou com o aumento da pressão numa relação quadrática, porém não atingiu um valor máximo, indicando que a pressão ideal não foi atingida no intervalo estudado; no entanto, elevou-se de 67 para $86 \%$, quando a pressão variou de 330 para $730 \mathrm{kPa}$ para v de $1 \mathrm{~m} \mathrm{~s}^{-1}$. Em intervalo de pressões mais usuais, de 500 a 730 $\mathrm{kPa}$ e v de $1 \mathrm{~ms}^{-1}$, o CUC variou de 77 para $86 \%$ e a influência da pressão sobre ele foi mais significativa, em termos práticos, que as variáveis VRA e DV.

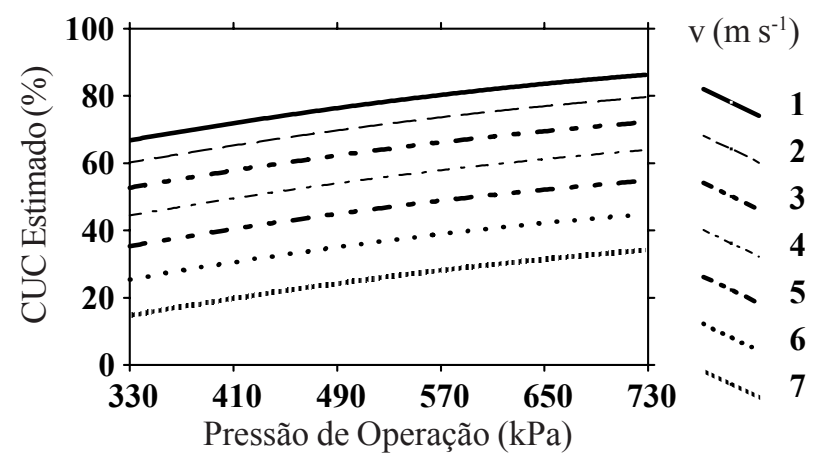

Figura 3. Estimativa do CUC em função da pressão de operação do aspersor para diferentes velocidades do vento, Ea de $72 \mathrm{~m}$, El de 84 m, DV de $90^{\circ}$ e VRA de 0,43 rpm, com distribuição entre aspersores retangulares

Velocidade do vento: Na Figura 4 (A) (B) e (C) são apresentadas as estimativas da variação do CUC em função da velocidade do vento, para diferentes espaçamentos entre aspersores (Ea), DV de $90^{\circ}$, VRA de 0,43 rpm, p de $686 \mathrm{kPa}$ e El de 96,84 e 72 m, respectivamente. 
(A)

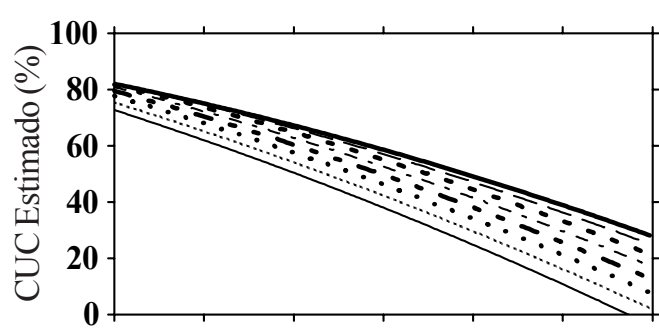

(B)

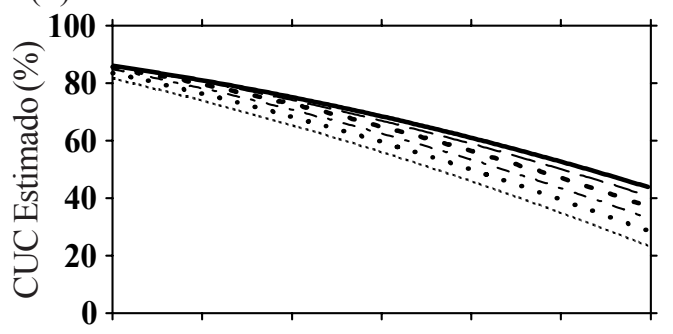

(C)

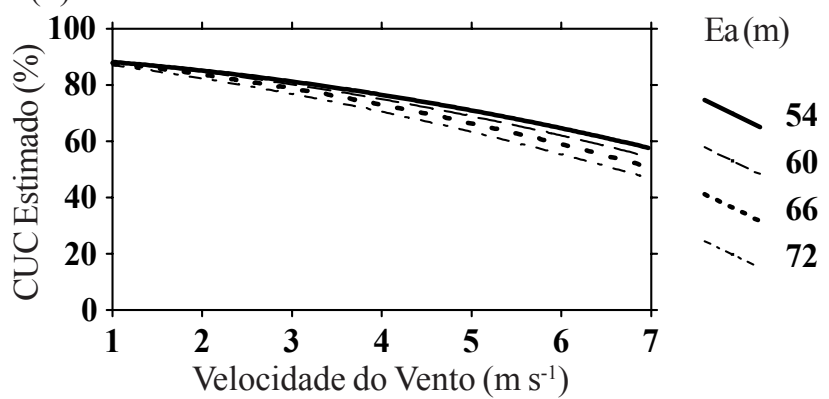

Figura 4. Estimativa do CUC em função da velocidade do vento para diferentes espaçamentos entre aspersores na linha lateral (Ea), El de $96 \mathrm{~m}$ (A), El de $84 \mathrm{~m}$ (B) e El de $72 \mathrm{~m}$ (C) DV de 90, VRA de $0,43 \mathrm{rpm}$ e p de $686 \mathrm{kPa}$, com distribuição entre aspersores retangulares

Para El de 96 m (Figura 4A) no espaçamento de 54 x 96 m (Ea x El) o CUC diminuiu de 82 para $28 \%$, quando a velocidade do vento aumentou de 1 para $7 \mathrm{~m} \mathrm{~s}^{-1}$, respectivamente; já para espaçamento de 96 × $96 \mathrm{~m}$, o CUC diminuiu de 73 para $0 \%$, quando v aumentou de 1 para $7 \mathrm{~m} \mathrm{~s}^{-1}$, respectivamente. Observa-se, também, que o CUC diminuiu em $9 \%$, quando Ea foi de 54 para $96 \mathrm{~m}$ com v de $1 \mathrm{~m} \mathrm{~s}^{-1}$ e de $28 \%$, quando Ea passou de 54 para $96 \mathrm{~m}$ com v de $7 \mathrm{~m} \mathrm{~s}^{-1}$. Com o aumento progressivo da velocidade do vento e do Ea, o CUC diminuiu. É interessante ressaltar que, para El de $96 \mathrm{~m}$, valores de CUC superiores ou iguais a $70 \%$ só foram obtidos para velocidades do vento menores que $2,7 \mathrm{~m} \mathrm{~s}^{-1}$, dependendo do valor de Ea utilizado. Quando El foi de $84 \mathrm{~m}$ (Figura 4B), observou-se que houve aumento geral do CUC em relação à Figura 4A, considerando-se as diferentes velocidades do vento; percebese, também, uma aproximação entre as diversas curvas de CUC para os diferentes EA, indicando que, para valores de EL, menores, a influência do Ea sobre o CUC diminui; para espaçamento de 54 por $84 \mathrm{~m}$ (Ea $\times \mathrm{El}$ ) o CUC variou de 86 para $44 \%$ com v entre $1 \mathrm{e}$ $7 \mathrm{~m} \mathrm{~s}^{-1}$, respectivamente e, no espaçamento de 84 por $84 \mathrm{~m}$, o CUC passou de 82 para $23 \%$, com v variando entre 1 e $7 \mathrm{~m} \mathrm{~s}^{-1}$, respectivamente. Valores de CUC iguais ou maiores que $70 \%$ só foram obtidos para $\mathrm{v}$ menores que $3,8 \mathrm{~m} \mathrm{~s}^{-1}$, dependendo do valor de Ea utilizado. Com a diminuição de El para 72 m (Figura 4C), houve aumento do CUC em relação aos El de 84 e 96 m; para espaçamento de 54 por $72 \mathrm{~m}$ (Ea x El) o CUC variou de 88 para $58 \%$, com velocidade do vento variando entre 1 e $7 \mathrm{~m} \mathrm{~s}^{-1}$, respectivamente, enquanto para o espaçamento de 72 por $72 \mathrm{~m}, \mathrm{o}$ CUC variou de 87 para $47 \%$, com a velocidade do vento se fixando em 1 e $7 \mathrm{~m} \mathrm{~s}^{-1}$, respectivamente. Para El de $72 \mathrm{~m}$, os valores CUC foram iguais ou maiores que $70 \%$ apenas para velocidades do vento menores que $5,0 \mathrm{~m} \mathrm{~s}^{-1}$, dependendo do valor de Ea utilizado.

\section{Aspersores em disposição triangular}

Velocidade de rotação do aspersor: $\mathrm{Na}$ Figura 5 são apresentadas as estimativas de CUC em função da velocidade de rotação do aspersor para diferentes velocidades do vento, observando-se que a VRA não teve forte influência sobre o CUC no intervalo estudado, apresentando uma variação máxima de $2,4 \%$ para uma dada velocidade do vento. Apresentou CUC máximo quando VRA atingiu $0,38 \mathrm{rpm}$, ou uma rotação a cada 2,63 min. Comparando-se os resultados apresentados nas Figuras 1 e 5, disposição entre aspersores retangular e triangular, respectivamente, observa-se que houve muito pouca diferença entre valores de CUC das duas figuras por influência do VRA e para pequenos valores de $\mathrm{v}\left(\mathrm{v}=1 \mathrm{~m} \mathrm{~s}^{-1}\right)$; entretanto à medida em que a velocidade do vento foi aumentando, a disposição triangular foi superando, de forma crescente a disposição retangular.

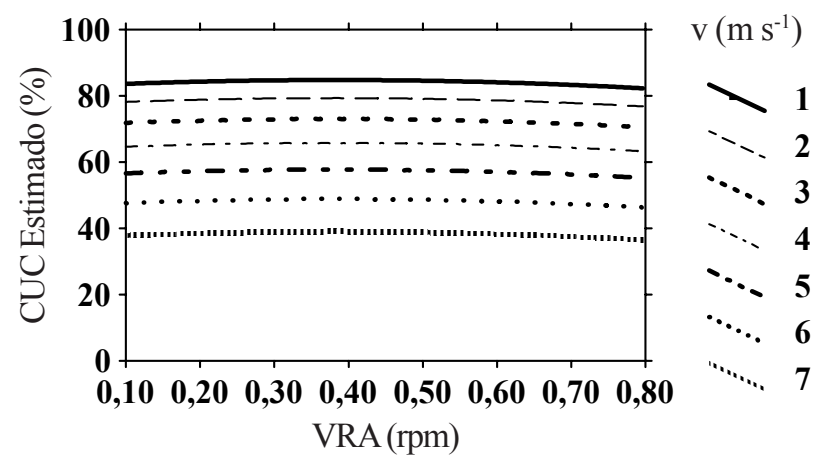

Figura 5. Estimativa do coeficiente de uniformidade de Christiansen em função da velocidade de rotação do aspersor para diferentes velocidades do vento, Ea de $72 \mathrm{~m}$, El de $84 \mathrm{~m}, \mathrm{DV}$ de $0^{\circ}$ e p de $686 \mathrm{kPa}$, com disposição entre aspersores triangulares

Direção do vento em relação à linha lateral: Tem-se, na Figura 6, as estimativas de CUC em função da direção do vento para diferentes velocidades do vento e disposição triangular entre aspersores. A direção do vento, tal qual ocorreu na disposição retangular entre aspersores (Figura 2) teve pequena influência sobre o CUC, apresentando uma diferença máxima entre valores extremos, de 1,38\%. O comportamento do CUC em função da DV na disposição triangular foi inverso ao encontrado na disposição retangular, ou seja, o CUC apresentou um ponto de mínimo quando o DV foi de $120^{\circ}$. A diferença entre os valores de CUC com DV de $120^{\circ}$ e $90^{\circ}$ foi de $0,09 \%$, ou seja, sem significado prático, indicando que, quando se utiliza disposição triangular entre aspersores, a linha lateral deve ser deixada, sempre que possível, paralela à direção do vento, cujo resultado ratifica os resultados encontrados por Azevedo (1998) trabalhando com aspersores de média pressão. 


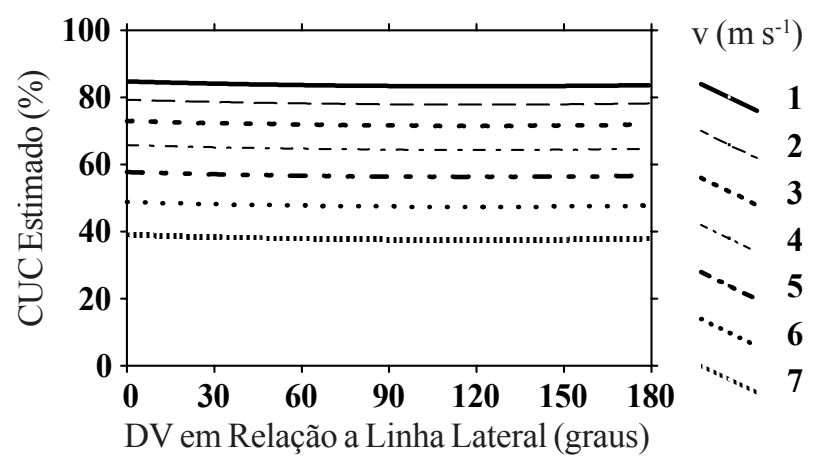

Figura 6. Estimativa do coeficiente de uniformidade de Christiansen em função da direção do vento em relação à linha lateral para diferentes velocidades do vento, Ea de $72 \mathrm{~m}$, El de 84 m, VRA de $0,38 \mathrm{rpm}$ e p de $686 \mathrm{kPa}$, com disposição entre aspersores triangulares

Pressão de operação do aspersor: Na Figura 7 são apresentadas as estimativas do CUC em função da pressão de operação do aspersor para diferentes velocidades do vento e aspersores em disposição triangular. O CUC aumentou de 67 para $86 \%$, quando a pressão aumentou de 330 para $730 \mathrm{kPa}$ e $\mathrm{v}$ se manteve em $1 \mathrm{~m} \mathrm{~s}^{-1}$. Tal qual a distribuição retangular entre aspersores, o CUC não atingiu um máximo com o aumento da pressão, indicando que a pressão ideal não foi atingida no intervalo pesquisado. Comparando-se os resultados apresentados nas Figuras 3 e 7, disposição entre aspersores retangular e triangular, respectivamente, observa-se que não houve, em termos práticos, diferença do CUC por influência da pressão, mas em relação à velocidade do vento observa-se que, a medida em que a mesma aumenta, cresce a diferença entre os valores do CUC, a favor da disposição triangular.

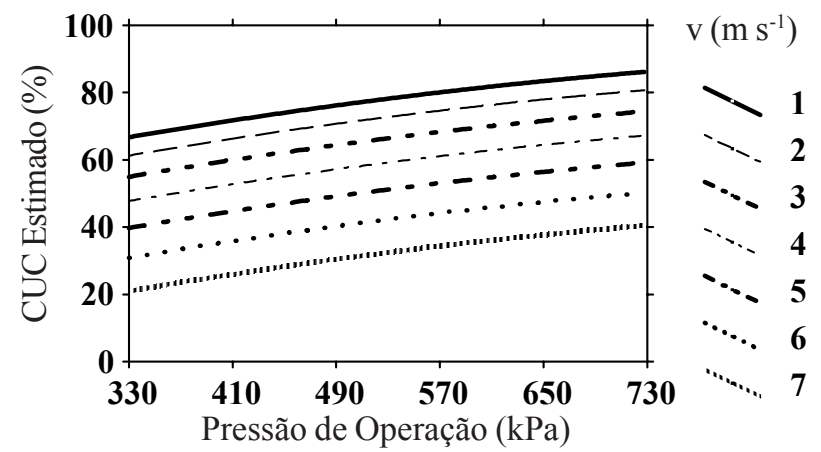

Figura 7. Estimativa do CUC em função da pressão de operação do aspersor para diferentes velocidades do vento, Ea de $72 \mathrm{~m}$, El de $84 \mathrm{~m}$, DV de $0^{\circ} \mathrm{e}$ VRA de $0,38 \mathrm{rpm}$, com disposição entre aspersores triangulares

Velocidade do vento: Na Figura 8A, B e C são apresentadas as estimativas da variação do CUC em função da velocidade do vento para diferentes espaçamentos entre aspersores (Ea), DV de $90^{\circ}$, VRA de $0,38 \mathrm{rpm}, \mathrm{p}$ de $686 \mathrm{kPa}$, distribuição triangular entre aspersores e El de 96, 84 e $72 \mathrm{~m}$, respectivamente.

Na Figura 8A o CUC diminuiu de 82 para $43 \%$, quando a velocidade do vento aumentou de 1 para $7 \mathrm{~m} \mathrm{~s}^{-1}$, respectivamente, para o espaçamento de $54 \times 96 \mathrm{~m}$ (Ea x El); já com espaçamento entre aspersores de $96 \times 96 \mathrm{~m}$, o CUC reduziu de 73 para $2 \%$, quando $\mathrm{v}$ se elevou de 1 para $7 \mathrm{~m} \mathrm{~s}^{-1}$, respectivamente.

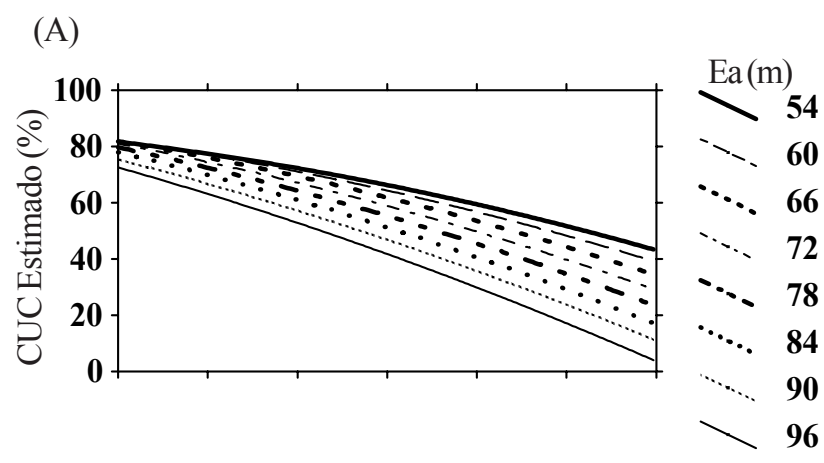

(B)

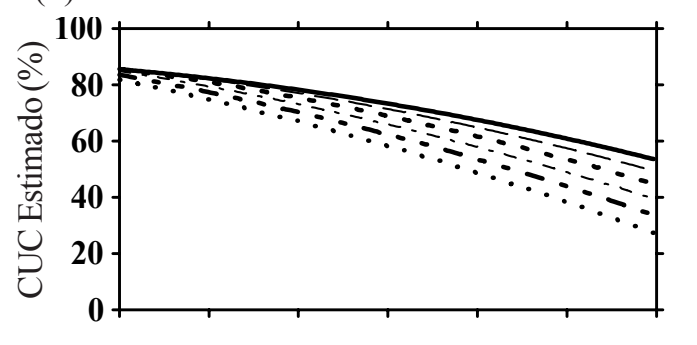

$\mathrm{Ea}(\mathrm{m})$

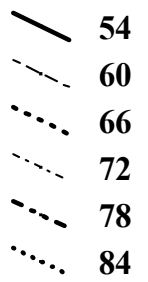

(C)

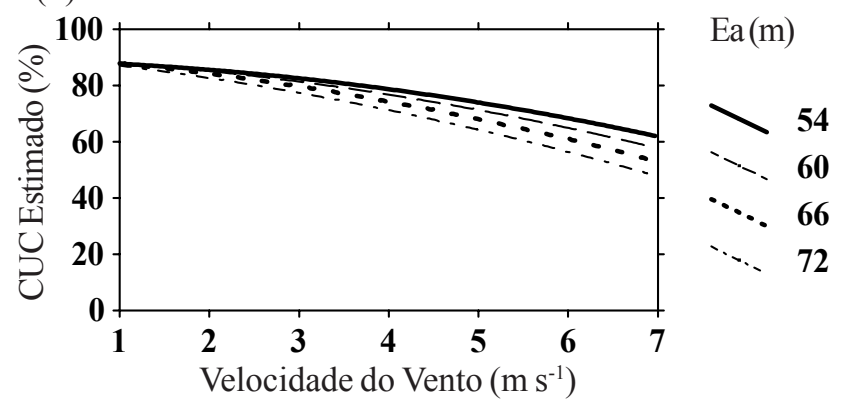

Figura 8. Estimativa do CUC em função da velocidade do vento para diferentes espaçamentos entre aspersores na linha lateral (Ea), El de $96 \mathrm{~m}$ (a), El de $84 \mathrm{~m}$ (b) e El de $72 \mathrm{~m}$ (c), DV de $0^{\circ}$, VRA de $0,38 \mathrm{rpm}$ e p de $686 \mathrm{kPa}$, com disposição entre aspersores triangulares

Comparando-se os valores apresentados nas Figuras 4A e 8A disposição entre aspersores retangular e triangular, respectivamente, observa-se que, para valores menores de $\mathrm{v}$ $\left(\mathrm{v}=1 \mathrm{~m} \mathrm{~s}^{-1}\right)$ não existe diferença, em termos práticos, entre valores de CUC, mas a medida em que a velocidade do vento aumenta, o CUC na disposição triangular (Figura 8A) vai superando, de forma crescente, a retangular. Para El de $96 \mathrm{~m}$, valores de CUC superiores ou iguais a $70 \%$ só foram alcançados para velocidades do vento menores que 3,3 $\mathrm{m} \mathrm{s}^{-1}$, dependendo do valor de Ea utilizado. Quando El diminui para $84 \mathrm{~m}$ (Figura 8B) para o espaçamento entre aspersores de 54 x $84 \mathrm{~m}$ (Ea x El) o CUC variou de 86 para 53\%, quando a velocidade do vento se elevou de 1 para $7 \mathrm{~m} \mathrm{~s}^{-1}$, respectivamente; já no espaçamento $84 \times 84 \mathrm{~m}$, o CUC passou de 82 para $27 \%$, quando a velocidade do vento se elevou de 1 para $7 \mathrm{~m} \mathrm{~s}^{-1}$, respectivamente. A diminuição do El de $96 \mathrm{~m}$ para $84 \mathrm{~m}$ resultou num aumento dos valores do CUC. Comparando-se os valores de CUC das Figuras 4B e 8B distribuição entre aspersores retangular e triangular, respectivamente, observa-se que, para valores menores de $\mathrm{v}\left(\mathrm{v}=1 \mathrm{~m} \mathrm{~s}^{-1}\right)$ não existe praticamente diferença; entretanto, a medida em que a velocidade do vento aumenta, o CUC da distribuição triangular supera os valores da retangular e com El de $84 \mathrm{~m}$, valores de CUC superiores ou 
iguais a 70\% só foram alcançados para velocidades do vento menores que 4,6 $\mathrm{m} \mathrm{s}^{-1}$, dependendo do valor de Ea utilizado. $\mathrm{O}$ aumento da velocidade do vento, de 1 para $7 \mathrm{~m} \mathrm{~s}^{-1}$, resultou numa variação do CUC de 88 para $62 \%$, com o uso de espaçamento entre aspersores de 54 × 72 m (Ea x El, Figura 8C). Utilizando-se o espaçamento de $72 \times 72 \mathrm{~m}$, o CUC variou de 87 para $48 \%$, quando $\mathrm{v}$ aumentou de 1 para $7 \mathrm{~m} \mathrm{~s}^{-1}$, respectivamente. $\mathrm{Na}$ distribuição triangular entre aspersores para El de $72 \mathrm{~m}$, os valores de CUC foram superiores aos da distribuição retangular (Figura 4C) similarmente como ocorreu nos casos anteriormente discutidos. Para El de $72 \mathrm{~m}$, valores de CUC superiores ou iguais a $70 \%$ só foram alcançados para velocidades do vento menores que $5,7 \mathrm{~m} \mathrm{~s}^{-1}$, dependendo do valor de Ea utilizado.

Considerando-se os intervalos das variáveis consideradas, a velocidade do vento foi o fator que mais influenciou a uniformidade de aplicação da água, seguida pela pressão de operação do aspersor, espaçamento entre aspersores na linha lateral, espaçamento entre linhas laterais, direção do vento em relação à linha lateral e velocidade de rotação do aspersor. Observou-se que, de forma geral, quando a velocidade do vento foi pequena $\left(\mathrm{v}<2 \mathrm{~m} \mathrm{~s}^{-1}\right)$ o espaçamento entre aspersores e entre laterais (Ea e El) não tiveram muita influência sobre o CUC, o que justifica o uso de maiores espaçamentos entre aspersores em horários de calmaria $\left(\mathrm{v}<0,5 \mathrm{~m} \mathrm{~s}^{-1}\right)$ e/ou em irrigações noturnas.

\section{CONCLUSÕES}

Considerando-se as estimativas de CUC, obtidas por meio dos modelos estatísticos desenvolvidos, conclui-se que:

1. Os CUC estimados diminuíram com o aumento da velocidade do vento e do espaçamento entre aspersores na linha lateral e entre linhas laterais, nos intervalos estudados $\left(0,53\right.$ a $7,17 \mathrm{~m} \mathrm{~s}^{-1}$ e 48 x $48 \mathrm{ma} 96$ x $96 \mathrm{~m}$ (Ea x El), respectivamente.

2. Os CUC estimados aumentaram com a elevação da pressão de operação, nos intervalos de valores estudados (318 a $729 \mathrm{kPa})$.

3. Os valores estimados dos CUC foram, de forma geral, maiores na disposição triangular entre aspersores que na retangular, igualando-se nas situações em que a velocidade do vento era baixa $\left(\mathrm{v}=1 \mathrm{~m} \mathrm{~s}^{-1}\right)$.

\section{REFERÊNCIAS BIBLIOGRÁFICAS}

ALI, S.M.A.; BAREFOOT, A.D. Low trajetory sprinkler patterns and evaporation loss. St Joseph, Michigam: ASAE, 1981. 24p. Paper, 81-2085

ALVES, A.D.; CASTRO, P.T. Desempenho de um sistema de irrigação por aspersão tipo canhão hidráulico, sob diferentes condições de velocidade de vento na região de Paracatu, CE, Brasil. Engenharia Rural, Piracicaba, SP, v.6, n.2, p.79-84, 1995.

ARRUDA, N.T. Análise de uniformidade de distribuição da água no sistema de irrigação por aspersão em linha. Viçosa: UFV, 1981. 80p. Dissertação Mestrado.

ASAE Procedure for sprinkler distribution testing for research purposes. In: Agricultural Engineers Yearbook. St Joseph, Michigan: ASAE, p.568-570, 1990.

AZEVEDO, H.J. Influência de elementos do clima no desempenho de sistemas de irrigação por aspersão na região Norte Fluminense. Viçosa, MG: UFV 1998.145p. Tese Doutorado.
BERNARDO, S. Manual de irrigação. 6. ed. Viçosa, MG: UFV, Imprensa Universitária, 1995. 596p.

CARVALHO, J.A. Tamanho de gotas e uniformidade de distribuição de água de dois aspersores de média pressão. Viçosa, MG: UFV, 1991. 73p. Dissertação Mestrado

CHARMELO, L.C.L. Desempenho de um equipamento de irrigação autopropelido, sob diferentes condições operacionais. Viçosa, MG: UFV, 1990. 50p. Dissertação Mestrado

CHRISTIANSEN, J.E. The uniformity of application of water by sprinkler systems. Agricultural Engineering, St Joseph, v.22, n.3, p.89-92, 1941.

CHRISTIANSEN, J.E. Irrigation by sprinkling. Berkely: University of California, Experiment Station, 1942. 124p. Bulletin, 670

GOMIDE, R.L; BERNARDO S.; VIEIRA M.; SEDIYAMA G.C. Modelo matemático para determinar a uniformidade de distribuição da água no sistema de irrigação por aspersão. Revista Ceres, Viçosa, MG, v.27, n.153, p.471-485, 1980.

KELLER, J. Sprinkler and trickle irrigation. Logan:Utah University, 1984. 621p.

MARTIN-BENITO, J.M.T.; GOMEZ, M.V.; PARDO, J.L. Working of sprinkler to optimize application of water. Journal of Irrigation and Drainage Engineering ASCE, New York, v.118, n.6, p.713-895, 1992.

MOYNAHAN, M.D. The use of sprinkler profiles to predict field performance. Logan Utah: USU, 1972. 79p. Masters Dissertation

NOGUEIRA, L.C.; SOUZA, F. Avaliação de dois sistemas de irrigação por aspersão II - Análise da uniformidade de distribuição. In: CONGRESSO BRASILEIRO DE ENGENHARIA AGRÍCOLA, 21, 1987, Anais..., Jundiaí: Sociedade Brasileira de Engenharia Agrícola, 1987. p.139.

PAZ, V.P. Perdas de água e uniformidade de distribuição na irrigação por aspersão. Viçosa, MG: UFV, 1990. 59p. Dissertação Mestrado

RIBEIRO J.M. Avaliação do sistema de irrigação por aspersão através do modelo linear. Fortaleza, CE: UFC, 1982. 89p. Dissertação Mestrado

RIBEIRO, J.M.; SOUZA, F. Avaliação da irrigação por aspersão: Influência do clima na uniformidade e eficiência. Ciência Agronômica, Fortaleza, v.14, n.1/2, p.123-136, 1983.

SHULL, H.; DYLLA, A.S. Traveling gun application uniformity in high winds. Transaction of the ASAE, St. Joseph, Michigan, v.19, n.2, p.254-258, 1976 a.

SHULL, H.; DYLLA, A.S. Wind effects on water application. Transaction of the ASAE, St. Joseph, Michigan, v.19, n.3, p.501-504, 1976 b.

SHULL, H.; DYLLA, A.S. Traveling boom sprinkler operation in wind. Transaction of the ASAE, St. Joseph, Michigan, v.22, n.3, p.537-539, 1979.

VORIES, E.D.; BERNUTH, R.D. Single nozzle sprinkler performance in wind. Transaction of the ASAE, St. Joseph, Michigan, v.29, n.5, p.1325-1330, 1986.

WIERSMA J.L. Effect of wind variation on water distribution from rotating sprinkler. Brooklings: South Dakota Agricultural Experiment Station, 1955. 18p. Bulletin, 16

ZOCOLER, J.L.; FRIZZONE, J.A.; PEREIRA, G.T.; PEREIRA, A.S. Uniformidade de distribuição de água versus custos do sistema de aspersão convencional. Engenharia Rural, Piracicaba, SP, v.6, n.2, p.39-48, 1995. 